\title{
Evidence of molecular alterations in the tumour suppressor gene $W W O X$ in benign and malignant bone related lesions of the jaws
}

\author{
MARINA GONÇALVES DINIZ1 ${ }^{1}$, ERICA RIEVRS BORGES ${ }^{1}$, \\ FLAVIO JULIANO PIMENTA ${ }^{1}$, ANA CAROLINA DE MESQUITA NETTO ${ }^{1}$, LUIZ DE MARCO ${ }^{2}$, \\ RICARDO SANTIAGO GOMEZ ${ }^{1}$ and CAROLINA CAVALIÉRI GOMES ${ }^{3}$ \\ Departments of ${ }^{1}$ Oral Surgery and Pathology, ${ }^{2}$ Surgery, and ${ }^{3}$ Pathology, \\ Universidade Federal de Minas Gerais, Av. Antonio Carlos, 6627 Belo Horizonte, MG Brazil, 31270901, Brazil \\ Received August 10, 2010; Accepted September 21, 2010
}

DOI: $10.3892 /$ or.2010.1094

\begin{abstract}
WWOX is a tumour suppressor gene altered in various human neoplasms. Deletion of WWOX is associated with bone metabolic defects and development of osteosarcoma in mice. We hypothesized that alterations of this gene are associated with the development of benign and malignant mesenchymal bone related lesions of the jaws. We investigated WWOX mRNA by nested reverse transcriptionPCR and direct sequencing and quantitative real-time PCR in two osteosarcoma, two fibrosarcoma, eight ossifying fibroma and two fibrous dysplasia fresh samples. Malignancy was associated with a decreased WWOX mRNA expression. Aberrant transcription pattern was found in five samples; however, the relative quantification (RQ) of the WWOX mRNA in such lesions was not different from those carrying only the wild-type. We provide new evidence of WWOX alterations in osteosarcomas and demonstrate for the first time alterations of this gene in fibrosarcomas as well as in ossifying fibromas of the jaws.
\end{abstract}

\section{Introduction}

Osteosarcoma is the most common non-hematologic bone malignancy in children and adults. Unlike other sarcomas, such as synovial sarcoma, alveolar rhabdomyosarcoma, and Ewing's sarcoma, no specific translocations or other genetic abnormalities have been identified in osteosarcoma (1). Loss of expression or mutations of the tumour suppressor genes retinoblastoma (RB1, MIM +180200), cyclin-dependent kinase inhibitor 2A (CDKN2A/TP16, MIM *600160), TP53 (MIM *191170), and activation of c-MYC, FOS, MDM2,

Correspondence to: Dr Carolina Cavalieri Gomes, Department of Pathology, Universidade Federal de Minas Gerais, Av. Antonio Carlos, 6627 Belo Horizonte, MG Brazil, 31270901, Brazil

E-mail: carolinacavalieri@yahoo.com

Key words: WWOX, osteosarcoma, fibrosarcoma, ossifying fibroma, fibrous dysplasia, benign fibro-osseous lesions among other genes have been described (1). However, the molecular pathogenesis of ostesarcoma remains to be established.

The fibrosarcoma, formerly a common subtype of softtissue sarcoma, is now a rather rare diagnosis, owing to advances in the classification of soft-tissue tumours (2). Although infantile fibrosarcoma shows a translocation $(12 ; 15)(\mathrm{p} 13 ; \mathrm{q} 26)$ that results in fusion of the NTRK3 gene (located on chromosome 15) to ETV6 gene (located on chromosome 12p), adult fibrosarcoma is not associated with specific translocations (3). Therefore, molecular changes related to fibrosarcomas are not fully understood.

Benign fibro-osseous lesions of the craniofacial complex comprise a diverse group of lesions characterized by replacement of bone by cellular fibrous tissue containing foci of mineralization that varies in amount and appearance (4). A member of this group is the fibrous dysplasia, a developmental condition arising as a result of activating missense mutations of the GNAS1 gene, that encodes the $\mathrm{GS} \alpha$ subunit of the heterotrimeric $\mathrm{G}$ protein complex $(5,6)$. It can lead to facial deformity/asymmetry in variable degrees. Ossifying fibroma and juvenile ossifying fibroma are benign neoplastic lesions of the jaws, which can cause bone expansion. The presence of clonal aberrations in a subset of ossifying fibroma and fibrous dysplasias cases has been demonstrated, suggesting that molecular alterations are responsible for such tumours (7). Indeed, we have previously demonstrated that mutations of the HRPT2 gene, a tumour suppressor gene, are associated with ossifying fibroma (8).

$W W O X$ is a tumour suppressor gene that spans the common fragile site FRA16D (9-11). The WWOX mutations are rare and usually the gene is altered by deletions or translocations in many cancer types including prostate, breast, esophageal, pancreatic, lung, stomach, and oral squamous cell carcinomas (10,12-18). Mice carrying a target deletion of the Wwox ${ }^{-/}$ gene developed osteosarcoma spontaneously (19). LudesMeyers et al (20) demonstrated that Wwox hypomorphic mice displayed reduced levels of Wwox. Although these animals had a shorter life span than the wild-type mice, they survived. These results suggest that low levels of Wwox protein are enough to overcome the lethality observed in the Wwox ${ }^{-/}$mice described by Aqeilan et al (21). In addition, a 
Table I. Diagnosis, gender and age of the patients of the samples included in the study.

\begin{tabular}{llll}
\hline Sample & Gender & Age & \multicolumn{1}{c}{ Diagnosis } \\
\hline 1 & F & 48 & Osteosarcoma \\
2 & F & 34 & Osteosarcoma \\
3 & M & 56 & Fibrosarcoma \\
4 & F & 51 & Fibrosarcoma \\
5 & F & 56 & Fibrous dysplasia \\
6 & F & 30 & Ossifying fibroma (primary) \\
7 & F & 33 & Ossifying fibroma (recurrence) \\
8 & F & 58 & Ossifying fibroma \\
9 & M & 15 & Ossifying fibroma \\
10 & M & 19 & Juvenile ossifying fibroma \\
11 & F & 24 & Ossifying fibroma \\
12 & F & 25 & Ossifying fibroma \\
13 & F & 28 & Ossifying fibroma \\
14 & M & 11 & Fibrous dysplasia \\
\hline
\end{tabular}

recent study in Wwox knock-out mice suggested that the Wwox has a significant role to bone tumour growth and the accompanying bone disease (21).

Considering all the available evidence regarding the importance of the WWOX in bone proliferation as well as the paucity of information regarding the expression of this gene in bone diseases involving the jaws, we conducted the present study.

\section{Materials and methods}

Human tissue. A total of 14 samples comprising two osteosarcomas, two fibrosarcomas, two fibrous dysplasia, seven ossifying fibromas and one juvenile ossifying fibroma were included in this study (Table I) according to criteria previously described (2). Five normal oral mucosa samples were also included as normal tissue reference. In each case, a portion of the lesion was resected, immediately snap frozen and stored at $-80^{\circ} \mathrm{C}$. For immunohistochemistry and histopathological analyses, the surgical samples were fixed in $10 \%$ buffered formalin and paraffin embedded. The local Ethics Committee approved the present study.

Quantitative real-time PCR ( $q P C R)$. Total RNA was isolated using Reagente Tri-Phasis (BioAgency, São Paulo, Brazil) and treated with DNase (Invitrogen Life Technologies, Carlsbad, CA, USA). cDNA was synthesized with Superscript FirstStrand Synthesis System Kit (Invitrogen Life Technologies) according to the manufacturer's instructions. For quantitative PCR analyses, wild-type transcripts of the WWOX cDNA were detected. We used 1X SYBR-Green PCR Master Mix (Applied Biosystems, Foster City, CA, USA) and primer sequences previously described (17). Reactions were performed in duplicate and ran on a Step One machine (Applied Biosystems). The cycling parameters were $10 \mathrm{~min}$ at $95^{\circ} \mathrm{C}$ followed by 40 cycles at $95^{\circ} \mathrm{C}$ for $15 \mathrm{sec}$ and $57^{\circ} \mathrm{C}$ for $1 \mathrm{~min}$
A

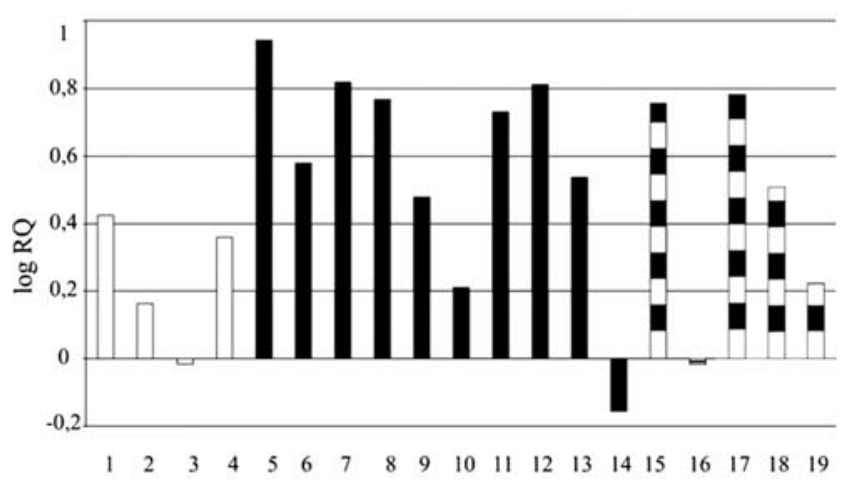

B

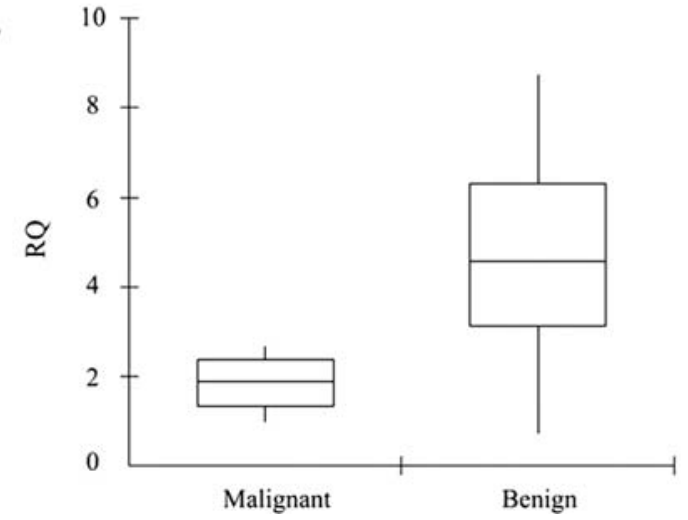

Figure 1. WWOX qPCR results. (A) Quantitative PCR showing higher transcription of the WWOX gene in malignant lesions (white bars), benign lesions (black bars) and normal oral mucosa (black and white bars) compared to normal blood (x-axis). Ostesarcoma (1 and 2), fibrosarcoma (3 and 4), fibrous dysplasia (5 and 14) and ossifying fibroma (6-13) and normal oral mucosa (15-19). (B) Despite expressing more WWOX than blood, decreased expression of WWOX was observed in malignant lesions (cases 1-4) compared to benign (cases 5-14) $(\mathrm{P}=0.047)$. RQ, relative quantification.

followed by melting curve analysis. WWOX expression was normalized to actin (internal control). The average threshold cycle $(\mathrm{Ct})$ for two replicates per sample were used to calculate $\triangle \mathrm{Ct}$. Relative quantification (RQ) of $W W O X$ cDNA was calculated with the $2^{-\Delta \Delta C t}$ method. As calibrator we used normal blood samples. Oral mucosa cDNA was used as normal tissue reference.

Nested reverse transcription-PCR (RT-PCR) and direct sequencing of transcripts. Total cDNA was used as a template for nested PCR amplification of the human WWOX cDNA as previously described (13). Glyceraldehyde-3-phosphate dehydrogenase $(G A P D H)$ cDNA was amplified as a control for cDNA quality. The amplified products were subjected to electrophoresis on a $6.5 \%$ polyacrylamide gel, followed by silver staining. cDNA bands corresponding to the normal and abnormal size transcripts were eluted and purified from agarose gel using the GFX PCR DNA and Gel Band Purification Kit (GE Healthcare, Buckinghamshire, UK) and sequenced. Briefly, 3-5 $\mu 1$ of purified PCR products were sequenced in both directions using two primer sets that amplify the whole open reading frame. DNA sequencing was performed on ABI PRISM 310 (Applied Biosystems) using the Big Dye terminator cycle sequencing kit (Applied 


$$
\text { A }
$$

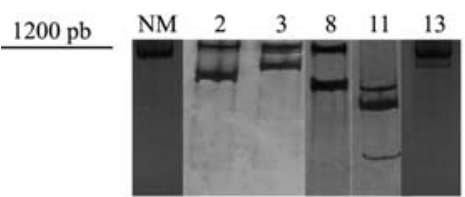

GAPDH

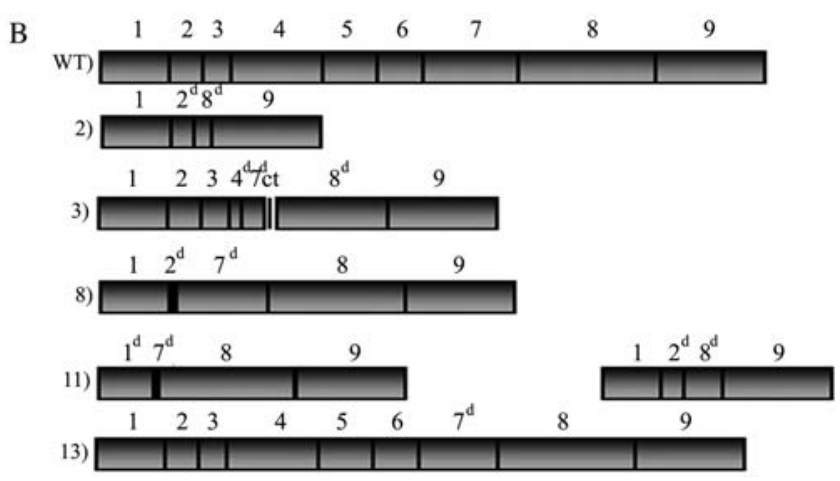

Figure 2. Analysis of $W W O X$ aberrant transcripts. (A) Total RNA was subjected to Transcriptase Reverse PCR (RT-PCR) and WWOX transcripts were visualized in silver stained $6.5 \%$ polyacrylamide gel. The WWOX wild-type (WT) band runs at approximately $1200 \mathrm{bp}$. GAPDH was used as control of cDNA synthesis. (B) Aberrant products were purified from gel bands and direct sequenced. NM, normal oral mucosa; 2, osteosarcoma; 3, fibrosarcoma; 8, 11 and 13, ossifying fibroma; indicates partial loss of the exon; ct, dinucleotide insertion. There was a polymorphism C969G located in the exon 8, nucleotide 969 (pro $\rightarrow$ ala), in the aberrant transcript of sample 13 .

Biosystems). GenBank accession number NM_016373.1 was used as a reference sequence.

Statistical analysis. Statistical significance was analysed with the Mann-Whitney test. $\mathrm{P}<0.05$ was considered to indicate statistical significance. These tests were performed with BioEstat software (Belém, Brazil), version 4.0.

\section{Results}

Quantitative PCR (qPCR) amplification was performed to determine only wild-type WWOX expression, as none of the aberrant transcripts conserved the sequences of qPCR primer annealing. As shown in Fig. 1A, on average, benign and malignant samples expressed more WWOX mRNA than the calibrator (blood of normal patients) (x-axis). However, there was a lower RQ (relative quantification) of WWOX mRNA in the malignant samples when compared to the RQ of the benign group $(\mathrm{P}=0.047)$, although the expression of both, malignant and benign groups, did not differ from the normal tissue control (Fig. 1).

As deletions are frequent in $W W O X$, in order to investigate the expression of aberrant WWOX mRNA, nested RT-PCR was carried out in all 14 tumour samples. Five samples (one osteosarcoma, one fibrosarcoma and three ossifying fibromas) showed aberrant transcripts with total or partial loss of exon length (Fig. 2). We found no statistically significant difference between the relative quantification (RQ) of the wild-type WWOX mRNA expression of samples showing aberrant products compared to those carrying only the wild-type
$(\mathrm{P}=0.62)$. An interesting finding when observing only the benign lesions was that the median of the RQ of WWOX mRNA in the samples carrying only the wild-type transcript (median 3.39) is similar to the RQ of the normal oral mucosa (median 3). On the other hand, the median of the RQ of the benign samples carrying aberrant transcripts (median 4.63) is higher than the RQ of the normal oral mucosa (median 3).

\section{Discussion}

In 2007, Aqeilan and colleagues (19) developed a mouse strain lacking WWOX expression. In the absence of any carcinogenic treatment, about $30 \%$ of juvenile mice developed focal lesions in the femur resembling early chondroid osteosarcomas. Histological examination suggested proliferation of progenitor cells arising from the periosteum. These authors concluded that the formation of these bone lesions supported the notion that, at least in the bone, $W W O X$ acts as a tumour suppressor. Two years after, Ludes-Meyers and colleagues (22) developed Wwox KO mice which presented bone metabolic defects. These animals showed smaller and thinner bones, having reduced bone volume as a consequence of a defect in mineralization, although no evidence of spontaneous neoplasia was observed in Wwox KO mice. In the present study we investigated the role of the WWOX gene in a set of human bone-related tumours.

First we investigated the transcription of the WWOX using quantitative real-time PCR (qPCR) reactions. Then, RT-PCR followed in order to investigate the presence of aberrant transcripts. The malignant samples showed a lower relative quantification of $W W O X$ compared to the benign samples (Fig. 1B). The data support the idea that WWOX works as a tumour suppressor gene in bone lesions as previously proposed by Aqeilan et al (19). The decreased expression levels of $W W O X$ in malignant neoplasias may be involved in the genetic regulation of many genes relevant to the malignant neoplasia pathogenesis, as WWOX interacts with other tumour suppressor proteins such as p53 and p73 $(24,25)$. The decreased WWOX expression we demonstrated in the malignant bone related tumor is in line with the recent findings of Kurek and colleagues, that showed absent or reduced expression of Wwox protein in 58\% of the ostosarcoma samples evaluated (28). In addition, a recent report using comparative genomic hybridization showed that the WWOX gene is deleted in three/ten osteosarcomas and also showed that the immunohistochemistry could not detect the protein in 34/55 samples (23).

The RT-PCR results demonstrated the existence of aberrant transcripts in one osteosarcoma, one fibrosarcoma and three ossifying fibroma samples. Whether this transcriptional alteration is an early event in the pathogenesis of such tumours or if it is just a result from the neoplasia progression remains to be elucidated. The biological role of such transcripts, if they have one, is speculative (26). As the WWOX gene is located in a fragile site, the aberrant transcripts are expected to be present in the neoplasias. Aqeilan and collegues (27) showed that tumours from $W W O X^{+/-}$mice demonstrated Wwox protein expression. Their results suggested that the inactivation of one allele of WWOX accelerates the predisposition of normal cells to malignant 
transformation (27). The presence of aberrant transcripts may be associated with reduced levels of the full length Wwox protein. Assuming that WWOX exhibits haploinsufficiency (27) the reduced expression of the wild-type protein could contribute to neoplasia development. In the present study, the presence of the aberrant transcripts did not associate with different $W W O X$ wild-type mRNA expression levels though. It is important to reinforce that the aberrant transcripts lacked the sequences of qPCR primers annealing, so that the qPCR detected only the normal size transcripts.

In conclusion, our study gives support to the notion that WWOX expression is altered in osteosarcomas and we provide additional evidence that it is also altered in fibrosarcomas. For the first time, we demonstrate the presence of WWOX aberrant transcripts in ossifying fibromas of the jaws. The meaning of such aberrant transcripts remains to be clarified.

\section{Acknowledgements}

This study was supported in part by grants from Fundação de Amparo à Pesquisa do Estado de Minas Gerais (FAPEMIG) and from Conselho Nacional de Desenvolvimento Científico e Tecnológico (CNPq), Brazil. Dr L. De Marco and R.S. Gomez are research fellows of CNPq.

\section{References}

1. Tang N, Song WX, Luo J, Haydon RC and He TC: Osteosarcoma development and stem cell. Clin Orthop Relat Res 466: 2114-2130, 2008.

2. Folpe AL: Soft-tissue tumours of the head and neck. In: Diagnostic Surgical Pathology of the Head and Neck. Gneep DR (ed). Elsevier, Philadelphia, pp660-663, 2009.

3. Sandberg AA: Cytogenetics and molecular genetics of bone and soft-tissue tumours. Am J Med Genet 115: 189-193, 2002.

4. Speight PM and Carlos R: Maxillofacial fibro-osseous lesions. Cur Diag Pathol 12: 1-10, 2006.

5. Weinstein LS, Shenker A, Gejman PV, Merino MJ, Friedman E and Spiegel AM: Activating mutations of the stimulatory G protein in the McCune-Albright syndrome. N Engl J Med 325: 1688-1695, 1991.

6. Schwindinger WF, Francomano CA and Levine MA: Identification of a mutation in the gene encoding the alpha subunit of the stimulatory $\mathrm{G}$ protein of adenylyl cyclase in McCuneAlbright syndrome. Proc Natl Acad Sci USA 89: 5152-5156, 1992.

7. Parham DM, Bridge JA, Lukacs JL, Ding Y, Tryka AF and Sawyer JR: Cytogenetic distinction among benign fibro-osseous lesions of bone in children and adolescents: value of karyotypic findings in differential diagnosis. Pediatr Dev Pathol 7: 148-158, 2004.

8. Pimenta FJ, Gontijo Silveira LF, Tavares GC, et al: HRPT2 gene alterations in ossifying fibroma of the jaws. Oral Oncol 42: 735-739, 2006

9. Bednarek AK, Laflin KJ, Daniel RL, Liao Q, Hawkins KA and Aldaz CM: WWOX, a novel WW domain-containing protein mapping to human chromosome 16q23.3-24.1, a region frequently affected in breast cancer. Cancer Res 60: 2140-2145, 2000 .
10. Ried K, Finnis M, Hobson L, et al: Common chromosomal fragile site FRA16D sequence: identification of the FOR gene spanning FRA16D and homozygous deletions and translocation breakpoints in cancer cells. Hum Mol Genet 9: 1651-1663, 2000.

11. Del Mare S, Salah Z and Aqeilan RI: WWOX: its genomics, partners, and functions. J Cell Biochem 108: 737-745, 2009.

12. Paige AJ, Taylor KJ, Taylor C, et al: WWOX: a candidate tumour suppressor gene involved in multiple tumour types. Proc Natl Acad Sci USA 98: 11417-11422, 2001.

13. Kuroki T, Trapasso F, Shiraishi T, et al: Genetic alterations of the tumour suppressor gene WWOX in esophageal squamous cell carcinoma. Cancer Res 62: 2258-2260, 2002.

14. Driouch K, Prydz H, Monese R, Johansen H, Lidereau R and Frengen E: Alternative transcripts of the candidate tumour suppressor gene, WWOX, are expressed at high levels in human breast tumours. Oncogene 21: 1832-1840, 2002.

15. Yendamuri S, Kuroki T, Trapasso F, et al: WW domain containing oxidoredutase gene expression is altered in nonsmall cell lung cancer. Cancer Res 63: 878-881, 2003.

16. Aqeilan RI, Kuroki T, Pekarsky Y, et al: Loss of WWOX expression in gastric carcinoma. Clin Cancer Res 10: 3053-3058, 2004.

17. Kuroki T, Yendamuri S, Trapasso F, et al: The tumour suppressor gene WWOX at FRA16D is involved in pancreatic carcinogenesis. Clin Cancer Res 10: 2459-2465, 2004.

18. Pimenta FJ, Gomes DA, Perdigao PF, et al: Characterization of the tumour suppressor gene WWOX in primary human oral squamous cell carcinomas. Int J Cancer 118: 1154-1158, 2006.

19. Aqeilan RI, Trapasso F, Hussain S, et al: Targeted deletion of Wwox reveals a tumour suppressor function. Proc Natl Acad Sci USA 104: 3949-3954, 2007.

20. Ludes-Meyers JH, Kil H, Nuñez MI, et al: WWOX hypomorphic mice display a higher incidence of B-cell lymphomas and develop testicular atrophy. Genes Chromosomes Cancer 46: 1129-1136, 2007

21. Aqeilan RI, Hassan MQ, de Bruin A, et al: The WWOX tumour suppressor is essential for postnatal survival and normal bone metabolism. J Biol Chem 283: 21629-21639, 2008.

22. Ludes-Meyers JH, Kil H, Parker-Thornburg J, et al: Generation and characterization of mice carrying a conditional allele of the Wwox tumour suppressor gene. PLoS ONE 4: e7775, 2009.

23. Yang J, Cogdell D, Yang D, et al: Deletion of the WWOX gene and frequent loss of its protein expression in human osteosarcoma. Cancer Lett 291: 31-38, 2010.

24. Chang NS, Doherty J, Ensign A, Schultz L, Hsu LJ and Hong Q: WOX1 is essential for tumour necrosis factor, UV light, staurosporine, and p53 mediated cell death, and its tyrosine 33-phosphorylated form binds and stabilizes serine 46-phosphorylated p53. Biol Chem 280: 43100-43108, 2005.

25. Aqeilan RI, Pekarsky Y, Herrero JJ, et al: Functional association between Wwox tumour suppressor protein and p73, a p53 homolog. Proc Natl Acad Sci USA 101: 4401-4406, 2004

26. Gourley C, Paije AJ, Taylor KJ, et al: WWOX mRNA expression profile in epithelial ovarian cancer supports the role of WWOX variant 1 as a tumour suppressor, although the role of variant 4 remains unclear. Int J Oncol 26: 1681-1689, 2005.

27. Aqeilan RI, Hagan JP, Aqeilan HA, Pichiorri F, Fong LY and Croce CM: Inactivation of the Wwox gene accelerates forestomach tumour progression in vivo. Cancer Res 67: 5606-5610, 2007.

28. Kurek KC, Del Mare S, Salah Z, et al: Frequent attenuation of the WWOX tumor suppressor in osteosarcoma is associated with increased tumorigenicity and aberrant RUNX2 expression. Cancer Res 170: 5577-5586, 2010. 